\section{AIDS virus and scrapie protein genes}

SIR-Haseltine and Patarca ${ }^{1}$ have suggested regions of sequence similarity between the 3'-end of the polymerase gene of HIV (human immunodeficiency virus, type HTLV-III) ${ }^{2}$ and the hamster gene for PrP 27-30, a protein associated with scrapie infectivity ${ }^{3,4}$, but Braun and Gonda have since shown that the protein sequence alignments are unconvincing 5 . Compiling scores by the NeedlemanWunsch-Sellars algorithm (program ALIGN $^{6}$ ) of sequence matches between the PrP gene and available lentiviral genes, including that of HTLV-III, we show here that the alignment of the DNA sequences is also without merit.

The regions of supposed nucleotide sequence similarity between $\operatorname{PrP}$ and HTLV-III are largely different from those for amino-acid sequences. Numerous gaps introduced into the DNA sequences for the purpose of alignment do not correspond to gaps in the protein sequence. Haseltine and Patarca's nucleic-acid alignment has four colinear regions of homology separated by regions of poor homology and different length. As in the case of protein alignment ${ }^{5}$, the number of gaps introduced lessens the alignment scores to the point where its significance is questionable. For example, alignment of the sequences of Fig. $1 a$ of Haseltine and Patarca ( $\mathrm{PrP}$ residues 207-631 and HTLV-III residues $4,632-5,123)$ with a low gap penalty of 2 (using the unitary scoring matrix of the PIR) and 100 randomizations, yields a score of 2.20 standard deviations $(\sigma)$. PrP aligned with the analogous visna virus ${ }^{7}$ and equine infectious anaemia virus $(\mathrm{EIAV})^{\mathrm{k}}$ regions gives $0.87 \sigma$ and $-0.22 \sigma$, respectively. For comparison, alignments of the HTLV-III region with the analogous (and evolutionarily related) regions of the visna virus and EIAV genomes gives scores of 8.91 and $9.74 \sigma$ at the same break penalty.

Using the program FASTN ${ }^{9}$ to screen the GENBANK files, scores similar to, or in excess of those raised by the lentiviralPrP alignments are frequent. For example, a section of the rat cytochrome P-450c gene $^{10}$ (residues 4,723-5,203) matches HTLV-III (residues $4,637-5,123$ ) with a score of $3.61 \sigma$. Finally, regions other than the polymerase gene in lentiviral genomes give high ALIGN scores when compared with the PrP gene sequence. Thus, residues 5,277-5,751 of HTLV-III score $3.27 \sigma$ when aligned to $\mathrm{PrP}$.

Haseltine and Patarca justify many of the gaps by the existence of small local (imperfect) repeats in the HTLV-III or PrP sequences. One of these 'repeats' is broken by the omission of the hexanucleotide GTGGAA following residue 261, an error which further extends a gap in the
HTLV-III sequence. Overall, the best patches of similarity occur in $\mathrm{G}-\mathrm{C}$ rich parts of the amino-terminal halves of the genes.

Thus, any relationships between the amino-acid or nucleic-acid sequences of HTLV-III and PrP are probably fortuitous.

J. Fernando Bazan

Department of Biophysics,

University of California,

Berkeley, California 94720, USA

Robert J. Fletterick

STANLEy B. PRUSINER

Departments of Biochemistry,

Biophysics and Neurology,

University of California,

San Francisco, California 94143, USA

1. Haseltine, W.A. \& Patarca, R. Nature 323, 115-116 (1986)

2. Ratner, L. et al. Nature 313, $277-284$ (1985).

. Oesch, B. et al. Cell 40, 735-746 (1985).

Basler, K. et al. Cell 46, 417-428 (1986).

. Braun, M.J. \& Gonda, M.A. Nature 325, 113-114 (1987).

. Needleman, S.W. \& Wunsch, C.D. J. molec. Biol. 48 $443-453(1970)$

7. Sonigo, P. et al. Cell 42, 369-382(1985).

8. Stephens, R.M., Casey, J.W. \& Rice, N.R. Science 231 $589-594(1986)$

9. Lipman, D.J. \& Pearson, W.R. Science 227, 1435-1441 (1985). 10. Sogawa, K. et al. Proc. natn. Acad. Sci. U.S.A. 81, 5066-
$5070(1984)$.

\section{Interferon- $\boldsymbol{\beta}_{2}$ living up to its name}

SiR-The B-cell differentiation factor BSF-2 (or BCDF), whose complementary DNA was recently cloned from $T$ cells by Hirano et al. ${ }^{1}$, turned out to be identical to interferon- $\beta_{2}$ (IFN- $\left.\beta_{2}, 26 \mathrm{~K}\right)$, which we had cloned from human fibroblasts by means of its interferon activity ${ }^{2-4}$. Billiau ${ }^{5}$, in pointing out this identity, questions the interferon-like function of the substance. We wish to recall the evidence which led us to classify this protein as an interferon and to consider the implications of these multiple activities.

Recombinant IFN- $\beta_{2}$ produced in $\mathrm{CHO}$ cells ${ }^{3}$ shows the following typical IFN activities. First, it inhibits vesicular stomatitis virus, Mengo virus, and herpes simplex virus- 2 in human fibroblasts and amniotic cells ${ }^{3,6}$. Second, it efficiently induces specific interferon-activated genes $^{3.4}$, such as $\left(2^{\prime}-5^{\prime}\right)$-oligo (A) synthetase and induces their messenger RNAs in the presence of cycloheximide, showing the action to be direct ${ }^{7}$. Third, it has antimitogenic effects on fibroblasts ${ }^{6}$. Fourth, there is species specificity ${ }^{3}$. And finally, IFN- $\beta_{2}$ displays activity on mousehuman hybrid cells containing human chromosome 21 , which carries the type I interferon receptor, and the activity is inhibited by antibodies to this receptor ${ }^{4.67}$. Both the antiviral activity of IFN- $\beta_{2}$ and its ability to induce $\left(2^{\prime}-5^{\prime}\right)$-oligo (A) synthetase are neutralized by several polyclonal and monoclonal antibodies to IFN $-\beta_{1}{ }^{3.7}$ (J. Vilcek and P.B. Sehgal, personal communication), but not by anti-

\section{Quantum jump}

SiR-Mention of our paper ${ }^{1}$ in a recent News and Views article ${ }^{2}$ leads me to emphasize that our experiments merely demonstrate a jumping time as short as about one second for the electron to complete the jump from the higher to the lower level. This is considerably shorter than the approximately 30 second dwell time in the upper electronic level, and the phenomenon therefore may legitimately be described as a 'jump'. It is not significantly shorter than our earlier example of a quantum jump $^{3}$ although our later experiments have employed a different technique ${ }^{4}$ to observe the jump, namely a new continuous type of Stern-Gerlach effect.

Department of Physics,

HANS DeHMELT

University of Washington,

Seattle, Washington 98195, USA

1. Negourney, W., Sandberg, J. \& Dehmelt, H. Phys. Rev.
Lett. 56, 2797 (1986).
2. Maddox, J. Nature 323, 577 (1986).
3. Van Dyck, R. Jr, Ekstrom, P. \& Dehmelt, H. Nature 262,
776 (1976).
4. Dehmelt, H. Proc. natn. Acad. Sci. U.S.A. 83, 2291; 3074
(1986).

bodies to IFN- $\alpha$ or IFN- $\gamma$, hence its designation as a $\beta$-type interferon. Structurally, IFN- $\beta_{2}$ has conserved half of the amino-acid residues common to all type I interferons ${ }^{3}$. But by its gene structure and localization, IFN- $\beta$ is only distantly related to the other interferons.

The finding of BSF-2 activity in an interferon molecule active on non-lymphoid cells, is not surprising in view of the known involvement of interferons in terminal differentiation ${ }^{3}$. With respect to B cells, IFN- $\alpha,-\beta$ and $-\gamma$ have been shown to induce chronic lymphocytic leukaemia (CLL) cells to acquire plasmacytoid characters with increased cytoplasmic immunoglobulin ${ }^{8}$. The growth of some CLL B cells is strongly stimulated by IFN$\alpha^{8}$, a finding that may be related to the plasmacytoma growth stimulation mentioned by Billiaus. IFN- $\gamma$ has been identified as a B-cell maturation lymphokine for normal murine B cells ${ }^{9}$ and, in the presence of interleukin-2, for human B cells ${ }^{10}$, producing strong increases in immunoglobulin secretion. Thus, interferons have Bcell differentiation effects.

IFN- $\beta_{2}$, like IFN- $\gamma$, is produced by $T$ cells, and both may exert lymphokine activity at concentrations lower than those giving antiviral effects ${ }^{11}$. Indeed, IFN- $\gamma$ induces HLA and anti-NK cell effects 100 times more efficiently than it inhibits viruses. IFN- $\beta_{2}$ has a specific activity $50-100$ times lower than IFN- $\beta_{1}$ against vesicular stomatitis virus ${ }^{2.3}$ but its antimitogenic activity on fibroblasts is 10 times higher than IFN- $\beta_{1}$ (unpublished data), supporting the proposed role ${ }^{12}$ of IFN- $\beta_{2}$ in fibroblast growth-control in response to 